\title{
Properties of New Parametric Generalized Entropy of Order Statistics
}

\author{
Rifat Nisa ${ }^{1^{*}}$ and M.A.K. Baig ${ }^{2}$ \\ ${ }^{1,2}$ P.G. Department of Statistics, University of Kashmir, Srinagar-190006, India
}

Available online at: www.isroset.org

Received: 01/Sept/2018, Accepted:11/Oct/2018, Online: 31/Oct/2018

\begin{abstract}
In this article, we introduce a new two parametric generalized uncertainty measure based on order statistics and also its residual version. Some general expressions of this proposed measure corresponding to particular probability distributions are derived. Finally, we study a lower bound for the proposed dynamic measure.
\end{abstract}

Keywords- Shannon's Entropy; Order Statistics; Probability Integral Transformation; Residual Entropy; Generalized Information.

\section{INTRODUCTION}

Shannon's [8] entropy is a significant concept introduced in information theory. It plays a decisive role in areas of engineering and physics as a measure of complexity and uncertainty in order to define and control many chaotic systems. The basic uncertainty measure is defined by Shannon for random variable (r.v.) $W$ as,

$H(W)=-\int_{0}^{\infty} f(w) \log f(w) d u=-E[\log f(w)]$

where $f(w)$ is the probability density function (pdf).

Assume that $W_{1}, W_{2}, \ldots, W_{n}$ be a random sample from a distribution function (cdf) $F(w)$ with pdf $f(w)$. By arranging $W_{1}, W_{2}, \ldots, W_{n}$ from the lowest to highest, the order statistics of the sample is defined as $W_{1: n} \leq W_{2: n} \leq \ldots \leq W_{n: n}$. The density of $k^{\text {th }}$ order statistics [1] is given by,

$$
f_{k: n}(w)=\frac{1}{B(k, n-k+1)}\left(F_{w}(w)\right)^{k-1}\left(1-F_{w}(w)\right)^{n-k} f_{w}(w)
$$

where

$$
B(u, v)=\frac{\Gamma u \Gamma v}{\Gamma(u+v)}
$$

Order statistics finds its application in the wide range of real world problems like robust statistical estimation, detection of outliers [3], description of probability distribution of record values and order statistics [2], casestudy of censored samples. Attributes of information pertaining to order statistics that are based on KullbackLeibler [7] and Shannon entropy [8] and measure using probability integral transformation have been thoroughly investigated by Ebrahimi et.al [6]. Two parametric generalized entropy, the Verma entropy [10] and [9] studied in context with order statistics. Various abstractions of Shannon's entropy [8] are present in the literature of information theory. There is a scope to further generalize the measure (1.1) in many ways and accordingly in this paper a new generalized Information measure is proposed.

In section II, we tend to specific generalized entropy of $K^{\text {th }}$ order statistics in terms of the generalized entropy of $K^{\text {th }}$ order statistics of exponential distribution and study a number of its properties. Section III, provide lower bound for entropy of order statistics. In section IV, we tend to derive an associate expression for residual generalized entropy of order statistics using residual generalized entropy for uniform distribution. In section $\mathrm{V}$, some characterized results are analyzed for the generalized residual entropy of the proposed measure.

\section{A NEW TWO PARAMETRIC GENERALIZED ENTROPY OF ORDER STATISTICS}

Presume $W$ be a continuous r.v. with pdf $f(w)$ and cdf $F(w)$, then the entropy of the type $(\theta, \phi)$ of the r.v. $W$ is given by,

$$
\begin{gathered}
H_{\theta, \phi}(W)=-\frac{\phi}{\theta-\phi} \log \int_{0}^{\infty} f^{\frac{\theta}{\phi}}(w) d w \\
\forall \phi \geq 1, \theta \neq \phi, 0<\theta<\phi
\end{gathered}
$$

where

$\lim _{\phi=1} H_{\theta, \phi}(W)=H_{\theta}(W)=\frac{1}{1-\theta} \log \int_{0}^{\infty} f^{\theta}(w) d w \quad$ is the Renyi's entropy 
and $\lim _{\phi=1, \theta \rightarrow 1} H_{\theta, \phi}(W)=-\int_{0}^{\infty} f(w) \log f(w) d w$ is the

Shannon entropy.

Using the substitution, $V=F(w)$ in (2.1) where $V$ has a standard uniform distribution. Let $V_{1}, V_{2}, \ldots, V_{n}$ be a random sample from a uniform distribution $[0,1]$ with the order statistics $z_{1} \leq z_{2} \leq \ldots \leq z_{n}$, then $z_{k}, k=1,2, \ldots, n$ has a beta distribution with pdf,

$$
\begin{gathered}
g\left(z_{k}\right)=\frac{1}{B(k, n-k+1)}\left(z_{k}\right)^{k-1}\left(1-z_{k}\right)^{n-k}, \\
0 \leq z_{k} \leq 1
\end{gathered}
$$

where

$B(u, v)=\int_{0}^{1} y^{u-1}(1-y)^{v-1} d y, u>0, v>0$ follows a beta distribution with $u$ and $v$ as parameters.

Now the generalized entropy of $W_{k: n}$ is represented as,

$H_{\theta, \phi}(W)=-\frac{\phi}{\theta-\phi} \log \int_{0}^{1} f^{\frac{\theta}{\phi}-1}\left(F^{-1}(V)\right) d V$

Table No.1: The expressions of GE for some lifetime

\begin{tabular}{|c|c|c|c|}
\hline Pareto & $\begin{array}{l}\frac{\omega \lambda^{\omega}}{w^{\omega+1}} \\
w \geq \lambda \\
\lambda>0 \\
\omega>0\end{array}$ & $\frac{\phi}{\phi-\theta} \log$ & $\left(\frac{\omega}{\lambda}\right)^{\frac{\theta}{\phi}} \frac{1}{\lambda\left(\frac{\theta}{\phi}(\omega+1)+1\right)}$ \\
\hline Weibull & $\begin{array}{l}\frac{1}{\lambda} e^{-\left(\frac{w-\omega}{\lambda}\right)} \\
w>\omega \\
\lambda>0 \\
\omega>0\end{array}$ & $\frac{\phi}{\phi-\theta}$ & {$\left[\log \left(\frac{\phi}{\theta \lambda^{\frac{\theta}{\phi}-1}}\right)\right]$} \\
\hline Lomax & $\begin{array}{l}\frac{\omega}{(1+w)^{\omega+1}} \\
w \geq 0 \\
\omega>0\end{array}$ & $\frac{\phi}{\phi-\theta} \operatorname{lo}$ & $\operatorname{og}\left[\frac{\omega^{\frac{\theta}{\phi}}}{\left(\frac{\theta}{\phi}(\omega+1)+1\right)}\right]$ \\
\hline
\end{tabular}

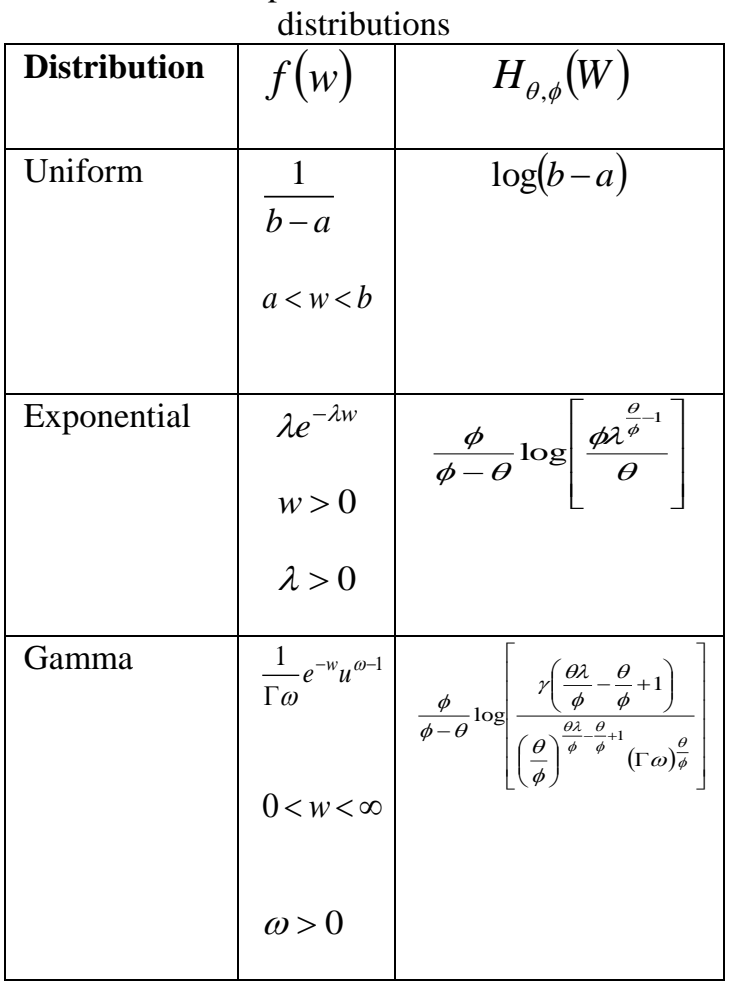

Theorem 2.1: The new two parametric generalized entropy of $w_{k: n}$ can be defined as,

$H_{\theta, \phi}\left(w_{k: n}\right)=H_{\theta, \phi}\left(z_{k}\right)$

$-\frac{\phi}{\theta-\phi} \log E_{g_{k}}\left[f^{\frac{\theta}{\phi}-1}\left(F^{-1}\left(X_{k}\right)\right)\right]$

where $H_{\theta, \phi}\left(z_{k}\right)$ denotes the entropy of beta distribution with parameters $k$ and $(n-k+1), E_{g_{k}}(w)$ denotes expectation of $w$ over $g_{k}$ and $X_{k} \sim g_{k}$ is the beta density with parameters $\left(\frac{\theta}{\phi}(k-1)+1\right)$ and $\left(\frac{\theta}{\phi}(n-k)+1\right)$.

Proof: We have

$$
\begin{aligned}
& H_{\theta, \phi}\left(z_{k}\right)=\frac{\theta}{\theta-\phi} \log B(k, n-k+1) \\
& -\frac{\phi}{\theta-\phi} \log B\left(\frac{\theta}{\phi}(k-1)+1, \frac{\theta}{\phi}(n-k)+1\right)
\end{aligned}
$$

Putting $z_{k}=F\left(w_{k: n}\right), k=1,2, \ldots, n$ which implies that $w_{k: n}=F^{-1}\left(z_{k}\right)$

Therefore, the generalized entropy of $w_{k: n}$ is given by, 


$$
\begin{aligned}
& H_{\theta, \phi}\left(w_{k: n}\right)=-\frac{\phi}{\theta-\phi} \log \int_{0}^{1} f^{-1}\left(F^{-1}\left(z_{k}\right)\right) \\
& \left(\frac{1}{B(k, n-k+1)}\left(z_{k}\right)^{k-1}\left(1-z_{k}\right)^{n-k} f\left(F^{-1}\left(z_{k}\right)\right)\right)^{\frac{\theta}{\phi}} d z_{k} \\
& =\frac{\theta}{\theta-\phi} \log B(k, n-k+1) \\
& -\frac{\phi}{\theta-\phi} \log B\left(\frac{\theta}{\phi}(k-1)+1, \frac{\theta}{\phi}(n-k)+1\right) \\
& -\frac{\phi}{\theta-\phi} \log \int_{0}^{1} \frac{\theta}{\phi}-1\left(F^{-1}\left(z_{k}\right)\right)\left(z_{k}\right)^{\frac{\theta}{\phi}(k-1)}\left(1-z_{k}\right)^{\frac{\theta}{\phi}}(n-k) \\
& \frac{1}{B\left(\frac{\theta}{\phi}(k-1)+1, \frac{\theta}{\phi}(n-k)+1\right)} d z_{k}
\end{aligned}
$$

Using (2.5) in (2.6) the required result (2.4) follows,

Example 2.1: Suppose $W$ is a r.v. that has an exponential distribution (ED) with pdf

$$
f(w)=\lambda e^{-\lambda w}, \lambda>0, w \geq 0
$$

Here $F(w)=1-e^{-\lambda w}$

Putting $X_{k}=F(w) \Rightarrow w=F^{-1}\left(X_{k}\right)$

$$
\begin{array}{r}
E_{g_{k}}\left[f^{\frac{\theta}{\phi}-1}\left(F^{-1}\left(X_{k}\right)\right)\right] \\
=E_{g_{k}}\left[f^{\frac{\theta}{\phi}-1}\left(-\frac{1}{\lambda} \log \left(1-X_{k}\right)\right)\right] \\
=\lambda^{\frac{\theta}{\phi}-1}\left(\frac{B\left(\frac{\theta}{\phi}(k-1)+1, \frac{\theta}{\phi}(n-k+1)\right)}{B\left(\frac{\theta}{\phi}(k-1)+1, \frac{\theta}{\phi}(n-k)+1\right)}\right)
\end{array}
$$

Putting $k=1$ in (2.5), we get

$$
\begin{aligned}
H_{\theta, \phi}\left(z_{1}\right) & =-\frac{\theta}{\theta-\phi} \log (n) \\
& +\frac{\phi}{\theta-\phi} \log \left(\frac{\theta}{\phi}(n-1)+1\right)
\end{aligned}
$$

Putting $k=1$ in (2.7), we get

$$
E_{g_{1}}\left[f^{\frac{\theta}{\phi}-1}\left(F^{-1}\left(X_{1}\right)\right)\right]=\lambda^{\frac{\theta}{\phi}-1}\left(\frac{\frac{\theta}{\phi}(n-1)+1}{n\left(\frac{\theta}{\phi}\right)}\right)
$$

Using (2.8) and (2.9) in (2.4), we have $H_{\theta, \phi}\left(W_{1: n}\right)=-\log (n)-\log (\lambda)+\frac{\phi}{\theta-\phi} \log \left(\frac{\theta}{\phi}\right)$

$$
\begin{gathered}
H_{\theta, \phi}(W)=-\frac{\phi}{\theta-\phi} \log \left(\frac{\phi}{\theta}\right)-\log (\lambda) \\
H_{\theta, \phi}\left(W_{1: n}\right)-H_{\theta, \phi}(W)=-\log (n) \\
H_{\theta, \phi}\left(W_{n: n}\right)=-\log (n)-\log (\lambda)-\frac{\phi}{\theta-\phi} \log \Gamma\left(\frac{\theta}{\phi}\right) \\
+\frac{\phi}{\theta-\phi} \log \left(\frac{\theta}{\phi}\right) \\
H_{\theta, \phi}\left(W_{n: n}\right)-H_{\theta, \phi}(W)=-\log (n) \\
-\frac{\phi}{\theta-\phi} \log \Gamma\left(\frac{\theta}{\phi}\right)
\end{gathered}
$$

\section{LOWER BOUND FOR THE NEW TWO PARAMETRIC GENERALIZED ENTROPY OF ORDER STATISTICS}

Theorem 3.1: A r.v. $W$ with $H_{\theta, \phi}(W)<\infty$ an entropy of the $k^{\text {th }}$ order statistics $W_{k: n}, k=1,2, \ldots, n$ is bounded below as,

$$
H_{\theta, \phi}\left(W_{k: n}\right) \geq H_{\theta, \phi}\left(z_{k}\right)-\log (M)
$$

where $M=f(m)<\infty, f$ is the pdf of $U$ and $m$ is the mode of the distribution.

Proof: The mode of the beta distribution $g_{k}$ is $m_{k}=\frac{k-1}{n-1}$

Thus, $g_{k}(X) \leq B_{k}=g_{k}\left(m_{k}\right)$

$=\frac{1}{B\left(\frac{\theta}{\phi}(k-1)+1, \frac{\theta}{\phi}(n-k)+1\right)}\left(m_{k}\right)^{\frac{\theta}{\phi}(k-1)}\left(1-m_{k}\right)^{\frac{\theta}{\phi}(n-k)}$

For $\phi>1,0<\theta<\phi$ from (2.4), we have

$$
\begin{gathered}
H_{\theta, \phi}\left(W_{k: n}\right) \geq H_{\theta, \phi}\left(z_{k}\right) \\
-\frac{\phi}{\theta-\phi} \log \int_{0}^{1} g_{k}\left(z_{k}\right)(M)^{\frac{\theta}{\phi}-1} d w_{k} \\
H_{\theta, \phi}\left(W_{k: n}\right) \geq H_{\theta, \phi}\left(z_{k}\right) \\
-\frac{\phi}{\theta-\phi} \int_{0}^{1} \frac{1}{B\left(\frac{\theta}{\phi}(k-1)+1, \frac{\theta}{\phi}(n-k)+1\right)}\left(z_{k}\right)^{\frac{\theta}{\phi}(k-1)} \\
\left(\left(1-z_{k}\right) \frac{\theta}{\phi}(n-k) \frac{\theta}{f^{\frac{\theta}{\phi}}-1}\left(m_{k}\right)\right) d z_{k} \\
H_{\theta, \phi}\left(W_{k: n}\right) \geq H_{\theta, \phi}\left(z_{k}\right)-\log (M)
\end{gathered}
$$

Example 3.1: Over the interval $[a, b]$ in the lifetime(uniform) distribution, we have $H_{\theta, \phi}(W)=\log (b-a)$ 


$$
\begin{aligned}
& H_{\theta, \phi}\left(z_{1}\right)=H_{\theta, \phi}\left(z_{n}\right)=-\frac{\theta}{\theta-\phi} \log (n) \\
& \quad+\frac{\phi}{\theta-\phi} \log \left(\frac{\theta}{\phi}(n-1)+1\right) \\
& H_{\theta, \phi}\left(W_{k: n}\right)-H_{\theta, \phi}\left(z_{k}\right) \geq \\
& -\frac{\phi}{\theta-\phi} \int_{0}^{1} \frac{1}{B\left(\frac{\theta}{\phi}(k-1)+1, \frac{\theta}{\phi}(n-k)+1\right)}\left(z_{k}\right)^{\frac{\theta}{\phi}(k-1)}\left(1-z_{k}\right)^{\frac{\theta}{\phi}(n-k)} f^{\frac{\theta}{\phi}-1}\left(m_{k}\right) \\
& H_{\theta, \phi}\left(W_{k: n}\right) \geq H_{\theta, \phi}\left(z_{k}\right)+\log (b-a)
\end{aligned}
$$

Substituting $k=1$ in above equation, we obtain

$H_{\theta, \phi}\left(W_{1: n}\right) \geq H_{\theta, \phi}\left(z_{1}\right)+\log (b-a)$

$H_{\theta, \phi}\left(W_{n: n}\right) \geq H_{\theta, \phi}\left(z_{n}\right)+\log (b-a)$

We conclude that the bounds for $H_{\theta, \phi}\left(W_{1: n}\right)$ and $H_{\theta, \phi}\left(W_{n: n}\right)$ are similar.

\section{RESIDUAL ENTROPY FOR THE NEW TWO PARAMETRIC GENERALIZED ENTROPY OF ORDER STATISTICS}

Ebrahim [5] for a random life time of $W$ of a system, at time $t$ the residual entropy is defined as,

$$
H(w ; t)=-\int_{t}^{\infty} f_{t}(w) \log f_{t}(w) d u
$$

Equation (4.1) evaluates the uncertainty in the remaining lifetime of the component which is believed to have survived to a lifetime of $t$.

where $f_{t}(w)$ is the pdf of the r.v. $W_{t}=(W-t / W>t)$ and is given by,

$f_{t}(w)=\left\{\frac{f(w)}{\overline{F(t)}}\right.$, if $w>t$

Using above result (4.1) can be rewritten as,

$H(w ; t)=-\int_{t}^{\infty} \frac{f(w)}{\bar{F}(t)} \log \frac{f(w)}{\bar{F}(t)} d u, t>0$

where $\bar{F}(t)=1-F(t)$ is the survival function (s.f.) of $W$. In the same way the generalized entropy of the residual life time $W_{t}$ is given by,

$$
\begin{gathered}
H_{\theta, \phi}(w ; t)=-\frac{\phi}{\theta-\phi} \log \int_{t}^{\infty}\left(\frac{f(w)}{\bar{F}(t)}\right)^{\frac{\theta}{\phi}} d w . \\
0<\theta<\phi, \phi \geq 1
\end{gathered}
$$

when $t=0$, it reduces to (2.1).

We note that the pdf and s.f. of $W_{k: n}$ (refer to [4] ), denoted by $f_{k: n}(w)$ and $\bar{F}_{k: n}(w), k=1,2, \ldots, n$ respectively are

$$
\begin{aligned}
& f_{k: n}(w) \\
& =\frac{1}{B(k, n-k+1)}(F(w))^{k-1}(1-F(w))^{n-k} f(w)
\end{aligned}
$$

where

$B(u, v)=\int_{0}^{1} y^{u-1}(1-y)^{v-1} d y, u>0, v>0 \quad, \quad$ follows beta distribution.

$$
\bar{F}_{k: n}(w)=\frac{\bar{B}_{F(w)}(k, n-k+1)}{B(k, n-k+1)}
$$

where

$$
B_{w}(u, v)=\int_{w}^{1} w^{u-1}(1-w)^{v-1} d w, 0<w<1
$$

follows, incomplete beta distribution.

Theorem 4.1: The new two parametric generalized residual entropy of the $k^{\text {th }}$ order statistics is expressed as $H_{\theta, \phi}\left(W_{k: n}, t\right)=H_{\theta, \phi}\left(z_{k}, F(t)\right)$

$$
-\frac{\phi}{\theta-\phi} \log E_{g_{k}}\left[f^{\frac{\theta}{\phi}-1}\left(F^{-1}\left(X_{k}\right)\right)\right]
$$

where $H_{\theta, \phi}\left(z_{k}, F(t)\right)$ represents the generalized residual entropy of the beta variate with parameters $(k \& n-k+1)$, $E_{g_{k}}(z)$ represents the expectation of $z$ over the random sample of size $n$ from uniform distribution on [0,1]. Then

$H_{\theta, \phi}\left(z_{k}, F(t)\right)=\frac{\theta}{\theta-\phi} \log \bar{B}_{F(t)}(k, n-k+1)$

$-\frac{\phi}{\theta-\phi} \log \bar{B}_{F(t)}\left(\frac{\theta}{\phi}(k-1)+1, \frac{\theta}{\phi}(n-k)+1\right)$

$g_{k} \& Z_{k} \sim g_{k}$ is the incomplete beta density with parameters $\left(\frac{\theta}{\phi}(k-1)+1, \frac{\theta}{\phi}(n-k)+1\right)$

Proof: Let $z_{k}$ be the $k^{\text {th }}$ order statistics based on represents the generalized entropy of beta variate with parameters $(k \& n-k+1)$.

$$
\begin{gathered}
H_{\theta, \phi}\left(W_{k: n}, t\right)=-\frac{\phi}{\theta-\phi} \log \left[\frac{\bar{B}_{F(t)}(k, n-k+1)}{B(k, n-k+1)}\right]^{\frac{\theta}{\phi}} \\
+\frac{\phi}{\theta-\phi} \log (B(k, n-k+1)) \frac{\theta}{\phi} \\
-\frac{\phi}{\theta-\phi} \log \int_{0}^{\infty}\left((F(w))^{k-1}(1-F(w))^{n-k} f(w)\right)^{\frac{\theta}{\phi}} d w
\end{gathered}
$$

Putting $X_{k}=F\left(W_{k: n}\right)$ we have $F^{-1}\left(X_{k}\right)=W_{k: n}$

$$
H_{\theta, \phi}\left(W_{k: n}, t\right)=\frac{\theta}{\theta-\phi} \log \bar{B}_{F(t)}(k, n-k+1)
$$




$$
\begin{aligned}
& -\frac{\phi}{\theta-\phi} \log \bar{B}_{F(t)}\left(\frac{\theta}{\phi}(k-1)+1, \frac{\theta}{\phi}(n-k)+1\right) \\
& -\frac{\phi}{\theta-\phi} \int_{F(t)}^{1} \frac{1}{B\left(\frac{\theta}{\phi}(k-1)+1, \frac{\theta}{\phi}(n-k)+1\right)}\left(X_{k}\right)^{\frac{\theta}{\phi}(k-1)}\left(1-X_{k}\right) \frac{\theta}{\phi}(n-k) f^{\frac{\theta}{\phi}-1}\left(F^{-1}\left(X_{k}\right)\right) d X_{k}
\end{aligned}
$$

using (4.7) in (4.8), we get the desired result (4.6).

Example4.1: Let $W$ be an exponentially distributed r.v. with pdf $f(w)=\lambda e^{-\lambda w}, w \geq 0, \lambda>0$, then $F(w)=1-e^{-\lambda w}$ putting $X_{k}=F(w)$

$E_{g k}\left(f^{\frac{\theta}{\phi}-1}\left(F^{-1}\left(X_{k}\right)\right)\right)$

$=\lambda^{\frac{\theta}{\phi}-1} \frac{\bar{B}_{F(t)}\left(\frac{\theta}{\phi}(k-1)+1, \frac{\theta}{\phi}(n-k+1)\right)}{\bar{B}_{F(t)}\left(\frac{\theta}{\phi}(k-1)+1, \frac{\theta}{\phi}(n-k)+1\right)}$

Putting $k=1$ (4.7) and (4.9), we get

$$
\begin{gathered}
H_{\theta, \phi}\left(z_{k}, F(t)\right)=\frac{\theta}{\theta-\phi} \log \left(\bar{B}_{F(t)}(1, n)\right) \\
-\frac{\phi}{\theta-\phi} \log \left(\bar{B}_{F(t)}\left(1, \frac{\theta}{\phi}(n-1)+1\right)\right) \\
E_{g_{1}}\left(f^{\frac{\theta}{\phi}-1}\left(F^{-1}\left(X_{1}\right)\right)\right)=\lambda^{\frac{\theta}{\phi}-1} \frac{\bar{B}_{F(t)}\left(1, \frac{\theta}{\phi} n\right)}{\bar{B}_{F(t)}\left(1, \frac{\theta}{\phi}(n-1)+1\right)}
\end{gathered}
$$

Using above results in (4.6), we get

$$
H_{\theta, \phi}\left(W_{1: n}, t\right)=-\log (n \lambda)+\frac{\phi}{\theta-\phi} \log \left(\frac{\theta}{\phi}\right)
$$

Also, $H_{\theta, \phi}(W, t)=-\frac{\phi}{\theta-\phi} \log \left(\frac{\phi}{\theta}\right)-\log (\lambda)$

Hence, $H_{\theta, \phi}\left(W_{1: n}, t\right)-H_{\theta, \phi}(W, t)=-\log n$

Therefore, the divergence in the above two discussed entropies is independent of time in the exponential case.

\section{PROPERTIES OF NEW TWO PARAMETRIC GENERALIZED RESIDUAL ENTROPY}

Theorem 5.1: Let $\bar{F}$ (survival function) be IGE (DGE) and $\phi>\theta$, then

$$
\begin{gathered}
\lambda_{F_{k: n}}(t) \leq(\geq)\left[\frac{\theta}{\phi} \exp \frac{(\phi-\theta)}{\phi} H_{\theta, \phi}\left(H_{\theta, \phi}\left(W_{k: n}, t\right)\right)\right]^{\frac{\phi}{\theta-\phi}} \\
t>0
\end{gathered}
$$

Proof: From (4.3), we have.

$$
\begin{aligned}
& \frac{(\phi-\theta)}{\phi} H_{\theta, \phi}^{\prime}\left(W_{k: n}, t\right) \\
& =\frac{\theta}{\phi} \lambda_{F_{k: n}}(t)-\frac{\lambda^{\frac{\theta}{\phi}} F_{k: n}(t)}{\exp \left[\frac{(\phi-\theta)}{\phi} H_{\theta, \phi}^{\prime}\left(W_{k: n}, t\right)\right]}
\end{aligned}
$$

Since $\bar{F}$ is IGE (DGE) and $\phi>\theta$,therefore, we have

$$
\begin{array}{r}
\lambda_{F_{k: n}}(t)\left[\frac{\theta}{\phi} \exp \left(\left[\frac{(\phi-\theta)}{\phi} H_{\theta, \phi}^{\prime}\left(W_{k: n}, t\right)\right]\right)\right. \\
\left.-\lambda^{\frac{\theta}{\phi}-1} F_{k: n}(t)\right] \geq(\leq) 0
\end{array}
$$

which leads to

$$
\begin{aligned}
& \lambda_{F_{k: n}}(t) \\
& \geq(\leq)\left[\frac{\theta}{\phi} \exp \frac{(\phi-\theta)}{\phi} H_{\theta, \phi}\left(H_{\theta, \phi}\left(W_{k: n}, t\right)\right)\right]^{\frac{\phi}{\theta-\phi}}
\end{aligned}
$$

Theorem 5.2: Let $W$ be the lifetime of a series system with pdf $f_{k: n}(w)$ and s.f. $\bar{F}_{k: n}(t), t>0$, then for $\phi>\theta$, the following un equivalence holds

$H_{\theta, \phi}\left(W_{k: n}, t\right) \geq H\left(W_{k: n}, t\right)$

Proof: We know that from $\log$ sum inequality,

$$
\begin{aligned}
& \int_{t}^{\infty} f_{k: n}(w) \log \frac{f_{k: n}(w)}{\left(\frac{f_{k: n}(w)}{\bar{F}_{k: n}}\right)^{\frac{\theta}{\phi}}} d w \\
& \geq\left(\int_{t}^{\infty} f_{k: n}(w) d w\right) \log \left(\frac{\int_{t}^{\infty} f_{k: n}(w) d w}{\int_{t}^{\infty}\left(\frac{f_{k: n}(w)}{\bar{F}_{k: n}}\right)^{\frac{\theta}{\phi}} d w}\right) \\
& =\bar{F}_{k: n}(t)\left(\log \bar{F}_{k: n}(t)-\frac{(\phi-\theta)}{\phi} H_{\theta, \phi}\left(W_{k: n}, t\right)\right)
\end{aligned}
$$

The LHS of (5.2) contributes to.

$\int_{t}^{\infty} f_{k: n}(w) \log f_{k: n}(w) d w+\frac{\theta}{\phi} \bar{F}_{k: n}(t) H\left(W_{k: n}, t\right)$.

Put (5.3) in (5.2), we obtain the desired result.

Table No. 2: Generalized residual entropy of some lifetime distributions 


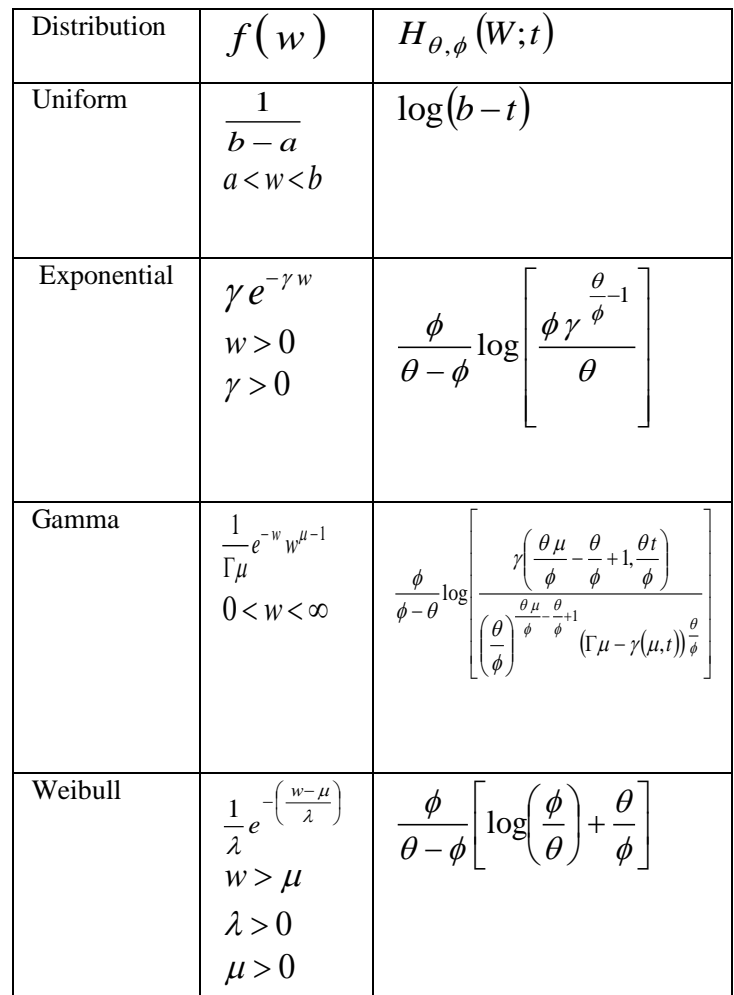
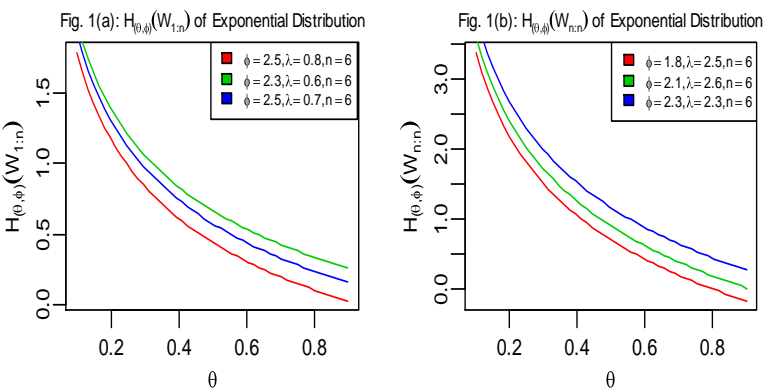

Fig.1: $H_{(\theta, \phi)}\left(W_{1: n}\right)$, and $H_{(\theta, \phi)}\left(W_{n: n}\right)$ plots of Exponential Distribution for $0.1 \leq \theta \leq 0.9$.

It is clear from Fig (1) that both the $H_{\theta, \phi}\left(W_{1: n}\right)$ and $H_{\theta, \phi}\left(W_{n: n}\right) \quad$ generalized entropies with respect to Exponential distribution are monotonic decreasing.

\section{CONCLUSION}

A new generalized two parametric entropy of order statistics is proposed in this paper. Various characterizations based on this measure are also studied. The generalized entropy finds its application in many practical situations like areas of engineering, physics etc. Some results of this new generalized entropy are also derived and the behavior of generalized expressions are mentioned graphically. The proposed two parametric measure can be of utmost research interest in future.

\section{REFERENCES}

[1]. B. C. Arnold, N. Blakrishan and N. H. Nagraja, "A First Course in Order Statistics", John Wiley and Sons, New York, 1992.

[2]. S. Baratpour, J. Ahmadi and N.R. Arghami, "Some Characterizations Based On Entropy of Order Statistics and Record Values", Communications in Statistics-Theory and Methods, Vol.36, Issue.1, pp.47-57, 2007.

[3]. J. Beirlant, E. J. Dudewicz, L. Gyorfi and E. C. Van Der Meulen, "Non Parametric Entropy Estimation: An Overview", International Journal of the Mathematical Statistics Sciences, Vol.6, Issue 1, pp.17-39, 1997.

[4]. H. A. David and H. N. Nagaraja, "Order Statistics," Wiley New York, 2003.

[5]. N. Ebrahimi, "How to Measure Uncertainty in the Residual Lifetime Distribution" Sankhya Series. A, Vol.58 pp.48-56, 1996.

[6]. N. Ebrahimi, E. S. Soofi and H. Zahedi, "Information Properties of Order Statistics and Spacing". IEEE Trans. Information Theory: Vol.50, pp.177-183, 2004.

[7]. S. Kullback, "Information Theory and Statistics", Wiley New York, 1959.

[8]. C. E. Shannon, "A Mathematical Theory of Communication". Bell System Technical Journal. Vol.27, Issue 3, pp.379423,1948.

[9]. R. Thapliyal, H.C. Taneja, "Generalized Entropy of Order Statistics" Applied Mathematics, Vol.3, pp.1977-1982, 2012.

[10]. R.S. Verma, "Generalization of Renyi's Entropy of Order $\alpha$," Journal of Mathematical Sciences, Vol.1, pp.34-48, 1966.

\section{Authors Profiles}

Rifat Nisa is a Ph.D Scholar in Department Of Statistics University Of Kashmir Srinagar (India). She received the B.Sc degree from Govt. Degree College for women Nawakadal Srinagar and received the Masters and $\mathrm{M}$. Phil in Statistics from Department Of

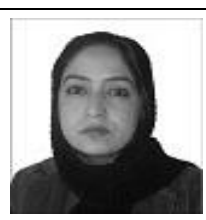
Statistics University Of Kashmir. From 2017 she is a research scholar of Ph.D in the Department Of Statistics University Of Kashmir Srinagar (India). Her research interest includes Information Theory, probability and order statistics.

M. A. K Baig completed his Graduation, Masters and Doctorial degree from A.M.U Aligarh (India). He was gold Medalist both in Graduation and Post-Graduation, currently he is working in University of Kashmir Srinagar (India), as Sr.Assistant

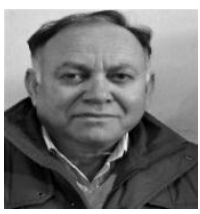
Professor. He has supervised Seven (7) Ph.D and Fifteen (15) M.Phil Scholars. He served as Head of the Statistics Department University of Kashmir Srinagar (India). His research interest includes Information Theory, Fuzzy Measures, Informatic Reliability. He has published more than 40 research papers in peer reviewed Journals. 